\title{
Monitoring System for Social Determinants of Health in Islamic Republic of Iran; Current Situation and Recovery Requirement
}

\author{
Ardeshir Khosravi 1,; Mariam Beheshtian ${ }^{2}$ \\ ${ }^{1}$ Deputy for Public Health, Ministry of Health and Medical Education and, Tehran, Iran \\ ${ }^{2}$ Genetics Research Center, University of Social Welfare and Rehabilitation Sciences, Tehran, Iran \\ * Corresponding author: Ardeshir Khosravi, Deputy for Public Health, Ministry of Health and Medical Education and, Tehran, Iran. Tel: +98-2181454211, E-mail: ardeshir.khosravi@ \\ gmail.com
}

Received: 22 Sep 2016

Accepted: 21 Nov 2016

Ppub: 23 Dec 2016

\begin{abstract}
Background: Social and political contexts of Islamic Republic of Iran (I.R. Iran) reflect strong commitment on reducing inequalities. This context provides a unique opportunity for reducing health inequalities mainly through targeting social elements. Methods: This paper discusses inter and intra sectoral activities adopted recent years to make a strategic planning in various domain of SDH and developing a monitoring system on social determinants of health in I.R. Iran. The paper is a narrative review of establishment and current situation of this monitoring system since 2005 within this system, the emphasis on health indictors has been shifted from overall calculation to the stratified which includes a wide range of determinants of both health services and leading causes of illness which may eventually result in health inequalities.

Results: Based on Strategic Planning 12 priority areas have been address to work on SDH. The system hires available infrastructures in health system as well as pertinent stakeholders. Furthermore, it provides a more comprehensive interdisciplinary means and recommendations for monitoring social elements to get better health equality. The set of indicators and their stratifiers presented in this study can serves as a good practice on addressing the social elements affecting health of Iranian population.

Conclusions: Based on revising of indicators for monitoring health inequality, 69 indicators have been approved by high council of health in 2016 and measuring of these indicators is a mandate for involved organizations in SDH planning.

Keywords: Health Equality; Iran; Social Determinants of Health; Monitoring System
\end{abstract}

